\title{
Steps to Better Cardiovascular Health: How Many Steps Does It Take to Achieve Good Health and How Confident Are We in This Number?
}

\author{
Catrine Tudor-Locke
}

Published online: 30 April 2010

(C) The Author(s) 2010. This article is published with open access at Springerlink.com

\begin{abstract}
Pedometers and other types of step-counting devices are growing in popularity with both researchers and practitioners. The focus of this article is on describing the most recent pedometer-related advances in terms of cardiovascular health. The emergent body of evidence suggests that pedometer-determined physical activity is related to a number of cardiovascular health outcomes and that intervention participants can realize modest changes in body mass index and blood pressure. Taking into consideration individual baseline values, tailored messages congruent with public health recommendations should promote incremental increases in steps/day on the order of an extra 3,000 to 4,000 (approximately $30 \mathrm{~min}$ ) of at least moderate intensity and taken in at least 10-minute bouts. Additional health benefits accrue with greater increases. Of course, even more benefits are possible from engaging in vigorous physical activity, but this seems less appealing for most people. Pedometer-based guidelines are not intended to supplant existing public health recommendations, but rather supplement them.
\end{abstract}

Keywords Pedometer - Accelerometer Physical activity . Walking

\section{Introduction}

With the release of its first-ever evidence-based physical activity guidelines report in 2008 , the US government

C. Tudor-Locke $(\square)$

Walking Behavior Laboratory,

Pennington Biomedical Research Center,

Baton Rouge, LA 70808, USA

e-mail: Tudor-Locke@pbrc.edu confirmed the very strong scientific evidence that continues to show that physically active people have higher levels of health-related fitness, a lower risk profile for developing a number of disabling medical conditions (including cardiovascular diseases), and lower rates of various chronic diseases than do people who are not active $[1 \bullet \bullet$. Further, they concluded that some physical activity is better than none, that additional health benefits accrue with greater volumes of physical activity, and that even more benefits may be had from participating in vigorous physical activity $[1 \bullet \cdot$. Because US adults average less than $2 \mathrm{~min} / \mathrm{d}$ of vigorous physical activity [2•], it stands to reason that the greater focus should be placed on increasing overall physical activity levels. Of all types of physical activity, walking stands out as the most popular form of leisure time exercise [3•], can be easily performed at moderate intensity [4], and is also a functional part of activities of daily living.

Armed with such information, clinicians and other practitioners represent frontline forces to motivate patients to realize the multiple and profound benefits of a physically active lifestyle. Among our arsenal, and growing in acceptance, is the simple and inexpensive pedometer, especially when imbedded as a motivational tool in an intervention program. In support of the efficacy of pedometer-based programming, at least two systematic reviews have catalogued positive effects on walking behaviors and some cardiovascular risk factors, including weight loss $[5 \bullet \bullet, 6 \bullet \bullet$ and systolic blood pressure [5••]. Clinical applications for pedometers include screening, prescription, monitoring, feedback, and evaluation. To nurture increased confidence in uptake of such technology, the focus of this article is to describe the most recent pedometer-related advances in terms of cardiovascular health. In particular, I attempt to lay the background information necessary to answer "how many steps does it 
take to achieve good health and how confident are we in this number?"

\section{How Are Steps Measured?}

Pedometers are typically waist-mounted instruments that vary in terms of cost and internal measurement mechanisms. There are generally two types of electronic pedometers on the market today: 1) a traditional version that detects steps taken using a spring-suspended horizontal lever arm, and 2) a newer version that contains a piezoelectric accelerometer. Both respond to vertical accelerations (ie, up and down motions) of the hip. The piezoelectric pedometers often have a memory storage feature (data from multiple days can be held in memory) and they may also offer an option to separately record only those steps that meet a minimum force threshold marketed as congruent with activity of at least moderate intensity. More advanced accelerometers such as those used in the National Health and Nutrition Examination Survey (NHANES) [7 ••] can provide additional information on time spent in different intensities of activity, but this usually is much more expensive technology that also requires

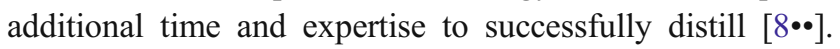
Additionally, these accelerometers are known to be sensitive to low-force accelerations [7••]. Subsequently, their inflated step output also represents erroneous steps and therefore needs to be manipulated to make them more comparable to commonly used research-grade pedometers (that are more likely to be used in clinical applications) $[7 \bullet \cdot]$. A more detailed comparison of different types of available instruments is published elsewhere $[8 \bullet \bullet$.

The most appropriate output from pedometers is the simple step taken [9]. In contrast, distance walked or energy expenditure are variables derived from manipulations of the raw step data, and these have been shown to produce additional layers of measurement error [10]. Pedometers recognize ambulatory activity, but their design (and that of other types of waist-mounted objective monitors) makes them unable to detect additional energy expended in climbing stairs, walking up hill, carrying loads, or other arm activities. Pedometers and other types of objective monitors cannot be used in water conditions (and therefore they cannot detect swimming) and they generally do not capture bicycling movements since the hips do not typically move up and down with enough force to generate step counts. Participation in these types of nonambulatory activities is relatively infrequent on a population level [3•]; however, when using pedometers for clinical purposes (eg, in interventions), a conversion factor to account for more frequent individual participation appears to be prudent [11].
Unfortunately, the various available instruments detect a "step" somewhat differently based on their own unique design and measurement properties. For example, sensitivity might be controlled by coiled or hair springs or internal microprocessors that innately differ between commercial products $[8 \cdot \bullet$. Regardless, outputs are reasonably comparable between studies and populations when high-quality, research-grade pedometers are used $[7 \bullet \bullet]$.

\section{Are Steps Related to Cardiovascular Health Outcomes?}

A systematic review assembled cross-sectional studies describing relationships between pedometer-determined step data and various cardiovascular risk factors. A weak inverse relationship was evident with both body mass index (BMI) and percentage overweight (median $r=-0.27$ and $r=$ -0.22 , respectively) [12]. Relationships with fitness ranged from weak to moderate depending on measure studied: 6-minute walk test (median $r=0.69$ ), timed treadmill test (median $r=0.41$ ), and estimated maximum oxygen uptake (median $r=0.22$ ). Self-reported participation in vigorous physical activity and accumulated steps/day has been independently and positively related to enhanced heart rate response to submaximal exercise [13]. More recently, Japanese researchers have developed a model to predict $\mathrm{VO}_{2}$ max in adult women from pedometer-determined steps/day, age, and BMI that correlates well $(r=0.81)$ with measured $\mathrm{VO}_{2} \max \left[1^{\bullet}\right]$. Schmidt et al. [15•] have demonstrated that individuals taking $\geq 5,000$ steps/day had substantially lower prevalence of adverse cardiometabolic health indicators than those taking lower amounts. Further, individuals taking $\geq 12,500$ steps/day had the best profiles compared with those taking lower amounts. Even in chronically ill populations, typically considered sedentary to low active, steps/day correlates with fitness, centrally distributed adiposity, and high-density lipoprotein cholesterol [16•]. Reduced pedometer-determined physical activity is a strong predictor of death in chronic heart failure, possibly surpassing traditional laboratory-based exercise tests [17]. Pedometer-determined physical activity has also been associated with better brachial artery flow-mediated dilation among individuals with peripheral arterial disease [18•].

As mentioned previously, systematic reviews have assessed the pedometer-intervention literature and have reported overall changes in steps/day $[5 \bullet \bullet, 6 \bullet \cdot]$, BMI $[5 \bullet \bullet$, $6 \bullet \bullet$, and blood pressure [5••]. Specifically, intervention participants increased their steps/day on average by approximately 2,500 [5••], achieved modest weight losses (approximately $0.05 \mathrm{~kg}$ per week on intervention) [6*•], and decreased their systolic blood pressure by $3.8 \mathrm{~mm} \mathrm{Hg}$ [5*0]. Most of these pedometer-based interventions have focused on relatively short-term changes in outcomes over 4 
to 16 weeks $[5 \bullet \bullet, 6 \bullet \cdot$, with the exception of two intervention studies that lasted 1 year or more [19, 20]. Sugiura et al.[20•] collected 2 years of pedometer data on 14 menopausal women who were instructed to attend a 90-minute physical education class once a week and increase their steps/day by 2,000 to 3,000 steps over baseline. Significant improvements were observed in serum lipids (total cholesterol, high-density lipoprotein cholesterol, and their ratio). Miyatake et al. [19] followed 31 Japanese men who were instructed to increase their daily steps. Visceral adipose tissue was reduced, and daily walking expressed as steps/day was found to be a greater predictor of its reduction compared with changes in exercise capacity at a 1-year examination. McTiernan et al. [21•] conducted a year-long exercise trial that used pedometers for measurement purposes (not for motivational purposes). They reported that participants who achieved the greatest incremental changes in steps/day assessed at 12 months from baseline also realized the greatest improvements in weight, hip circumference, BMI, body fat, and intra-abdominal fat. There is a strong negative relationship between study duration and resulting weight loss $[6 \bullet \bullet$, indicating that benefits are potentiated with prolonged adherence.

\section{How Many Steps Do People Normally Take?}

In terms of normative data, recent US estimates indicate that typical modern adult values are between 5,900 and 6,900 steps/day [7••, 22, 23]. In contrast, traditional Amish women and men (who reject a modern lifestyle) take 14,000 to 18,000 steps/day [24]. The stark discrepancy in these two sources of normative data suggest that contemporary living negatively impacts physical activity levels, but it does not clearly answer dose-response questions.

\section{How Many Steps Are Equivalent to Public Health Recommendations?}

Public health recommendations state that, for substantial health benefits, adults should accumulate $150 \mathrm{~min} / \mathrm{wk}$ of at least moderate intensity activity, or $75 \mathrm{~min} / \mathrm{wk}$ of vigorous intensity activity. It is possible to translate these recommendations in terms of pedometer-determined step data. A cadence of approximately 100 steps per minute is considered the floor value for moderate intensity activity (ie, at least 3.0 metabolic equivalents [METs]) [25••, 26], and approximately 130 steps per minute is congruent with vigorous intensity activity (ie, at least 6.0 METs) [26]. Using simple arithmetic, these time-based recommendations can be translated as 15,000 steps per week in moderate-intensity activity (or 3,000 steps/day for $30 \mathrm{~min}$ for 5 days) or 9,750 steps per week (or 3,250 steps/day for $25 \mathrm{~min}$ for 3 days) in vigorous-intensity activity. To be truly consistent with public health recommendations, however, these steps should be accumulated in bouts of at least $10 \mathrm{~min}$ and preferably spread out throughout the week [27••]. Importantly, the unstated implication is that these steps should be taken over and above some otherwise undeclared baseline level of activity.

If we consider normative data (ie, 5,900-6,900 steps/ day) as indicative of baseline levels of activity, then simply adding step-based translations results in a value of 8,900 to 9,900 steps/day (to be taken over 5 days in 1 week, for example), of which at least 3,000 should meet moderate intensity and bout requirements. Applying the same logic, achieving 9,150 to 10,150 steps/day on at least 3 days of the week by adding at least 3,250 steps/day taken at vigorous intensity (and in at least $10 \mathrm{~min}$ bouts) also conforms to public health recommendations. Combinations of moderate and vigorous intensity performed at varying frequencies during the week provide endless opportunities for accumulating healthy physical activity. The advantage of such multiple options is that they fit well within accepted theoretical notions underlying approaches to individualize successful behavior change [28].

Studies that have concurrently collected data on time in intensity and steps have shed light on the relationship between these two outputs. For example, a recent interpretation of NHANES accelerometer data using pedometerbased scales indicated that those who achieve between 7,500 and 9,999 steps/day also average approximately 30 min of moderate-to-vigorous physical activity, and those who achieve between 10,000 and 12,499 steps/day average approximately $60 \mathrm{~min}$ of moderate-to-vigorous physical activity $[29 \bullet \bullet$. As additional confirmation, a dose-response study in postmenopausal women demonstrated that those who averaged approximately 7,500 steps/day achieved the equivalent of public health recommendations for physical activity energy expenditure [30]. As steps/day increases, time spent in activity that is of at least moderate intensity also increases; a measure of pedometer-determined steps/ day explains approximately $63 \%$ of time spent in moderateintensity activity [29••]. The reason this cannot also be said of vigorous activity is only because this behavior is so rare on the population level $[2 \bullet, 29 \bullet \bullet]$. However, on an individual level, exercise remains a prominent strategy for increasing steps/day [31].

\section{Is There a Simpler Way of Conveying Step Guidelines for Physical Activity?}

Obviously the combinations and permutations of volume, intensity, days of the week, bout length, and so forth can 
become cumbersome when trying to provide clear guidance to patients. In 2004, Tudor-Locke and Bassett Jr. [32] established preliminary pedometer-determined physical activity cut-points for healthy adults: 1) $<5,000$ steps/day (sedentary); 2) 5,000 to 7,499 steps/day (low active); 3) 7,500 to 9,999 steps/day (somewhat active); 4) $\geq 10,000$ to 12,499 steps/day (active); and 5) $\geq 12,500$ steps/day (highly active). These categories were reinforced in 2008 [33••], and in 2009 the original sedentary level was further spliced into two additional levels: $<2,500$ steps/day (basal activity) and 2,500 to 4,999 steps/day (limited activity) [7••]. This hierarchical approach (Fig. 1) to presenting pedometerdetermined physical activity also fits within motivational stage-based schemes by considering individual baseline levels of activity and conveying the dose-response nature of participating in incrementally more and more physical activity. Each of these mounting doses represents a 2,500 -step increment over the previous dose. It is approximately equal to 20 to $25 \mathrm{~min}$ of physical activity [26] and is the amount that pedometer-based interventions typically elicit [5••]. Expressed another way, and considering, for example, a baseline of 5,000 steps/day, 7,500 steps/day would represent a 50\% increase, and 10,000 steps/day a $100 \%$ increase. As noted previously, some physical activity is better than none, and additional health benefits accrue with greater volumes of physical activity $[1 \bullet \bullet]$. Depending on the specific outcome of interest, evidence suggests that the greatest "bang for the buck" occurs when motivating the most inactive individuals to increase their physical activity by even a single stage [34]. Diminishing relative returns would be expected with similar absolute increments added to higher levels of activity. Of course, at all stages, everyone should be encouraged to take these extra steps at

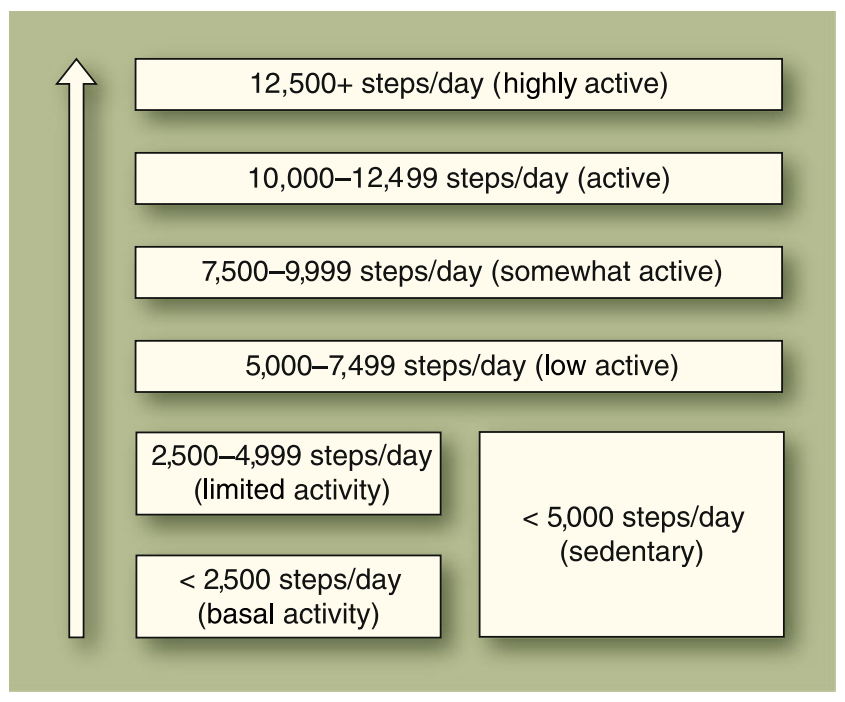

Fig. 1 Step-defined physical activity hierarchy least moderate intensity and in minimal 10-minute bouts. Even more benefits are possible by supplementing with vigorous physical activity $[1 \bullet \bullet]$.

\section{How Confident Are We About All This?}

There are a number of things I am concerned about with regard to objective monitoring of physical activity as a whole [35•]. Objective monitors, whether pedometers or accelerometers, are commercial items and favorite models disappear and new ones appear sporting additional valueadded features at regular intervals. This makes comparison between studies and populations using different instrumentation challenging. Further, although manufacturers may claim that new models are "fully backwards translatable," contrary evidence is emerging [36•, 37•]. Technological advances should be embraced, but measurement standardization would assure us that what is detected as a step today will remain so into the future. Unfortunately, manufacturers' measurement technology is patent-protected and this forces competitors to develop alternative approaches, so a solution to the problem is not readily at hand. There is no authoritative body at this time that requires standardization of outputs or otherwise oversees quality control. The ramification is that what is labeled a step by one instrument is not necessarily the same thing as another instrument. The measurement of steps is not the only output affected; the same goes for objectively monitored time spent in moderate-to-vigorous physical activity and sedentary behaviors as well. Further, the race to market increasingly sensitive instrumentation produces an inevitable specificity tradeoff that results in the detection of more spurious steps. Such heightened sensitivity undermines efforts to focus on the benefits of higher-intensity walking. The fact that research grade pedometers "fail" to detect very slow steps can actually be considered a design feature that can be used to effectively prod users to walk at higher speeds [38••].

I will be the first to argue that we should not be interpreting a "step" overly precisely. It is difficult to detect differences in individuals who differ by less than 500 steps/ day (approximately equivalent to a 5-minute difference in activity) and possibly by less than 1,000 steps/day. It is more appropriate to speak in terms of step ranges. So, 3,000 to 4,000 steps is more realistic in terms of communicating the amount taken in $30 \mathrm{~min}$ of moderate-intensity walking, allowing for individual variation $[25 \bullet \cdot, 26]$. And 7,500 to 9,999 steps/day corresponds to a category labeled as somewhat active $[32,33 \cdot 0]$ that represents increasing the likelihood of achieving minimal public health requirements for physical activity [7••, 30, 39].

Current public health recommendations are based on years of accumulated evidence. Pedometer-based guidelines 
are not intended to supplant this knowledge but rather supplement it. Pedometers are simply another tool in our arsenal. However, like all tools, knowledge of how best to use them is imperative and can optimize their effectiveness. The growing knowledge base includes recent systematic reviews $[5 \bullet \bullet, 6 \bullet \bullet$ that provide expected change data necessary for comparison and interpretation purposes, updates on methodologic considerations useful for guiding screening and monitoring [8••], and an assemblage of behavioral factors related to successfully increasing physical activity in pedometer-based interventions [38••].

\section{Conclusions}

In summary, promoting increased walking behaviors is consistent with public health recommendations. Pedometers and other step counters are useful tools that can be used for screening, prescription, monitoring, feedback, and evaluation purposes. The emergent body of evidence suggests that pedometer-determined physical activity is related to a number of cardiovascular health outcomes. In particular, intervention participants can realize modest changes in BMI and blood pressure with increases of physical activity. Further, continued participation appears to elicit accrued benefits. US adults currently take approximately 5,900 to 6,900 steps/day. Taking this as indicative of baseline levels, step-based translations of public health recommendations suggest that US adults should be taking 8,900 to 9,900 steps/day on 5 days of the week for example, at least 3,000 of which should meet moderate intensity and bout requirements. Alternatively, they can also meet guidelines by achieving 9,150 to 10,150 steps/day on at least 3 days of the week by adding at least 3,250 steps/day taken at vigorous intensity and in at least 10 -minute bouts. Because baseline levels of physical activity actually vary more widely in individuals, tailored messages should promote incremental increases in steps/day on the order of 3,000 to 4,000 extra steps/day (approximately $30 \mathrm{~min}$ ), again of at least moderate intensity and in at least 10-minute bouts. Not only is this increment congruent with public health guidelines, it also encourages moving up at least one stage, if not two, when applied to the stage-based pedometer step hierarchy described here. Of course, even more benefits are possible from engaging in vigorous physical activity, but this seems less appealing for most people. Pedometerbased guidelines are not intended to supplant existing public health recommendations, but rather supplement them.

Disclosure No potential conflict of interest relevant to this article was reported.
Open Access This article is distributed under the terms of the Creative Commons Attribution Noncommercial License which permits any noncommercial use, distribution, and reproduction in any medium, provided the original author(s) and source are credited.

\section{References}

Papers of particular interest, published recently, have been highlighted as:

- Of importance

•- Of major importance

1. •- Physical Activity Guidelines Advisory Committee: Physical Activity Guidelines Advisory Committee Report, 2008. Washington, DC: U.S. Department of Health and Human Services; 2008. Available at http://www.health.gov/paguidelines/committeereport. aspx. This report reivews existing scientific evidence to support recommendations for physical activity. This document was released in tandem with the first-ever federal physical activity guidelines.

2. - Troiano RP, Berrigan D, Dodd KW, et al.: Physical activity in the United States measured by accelerometer. Med Sci Sports Exerc 2008, 40:181-188. This cross-sectional analysis of the National Health and Nutrition Examination Survey (NHANES) accelerometer data reports time spent in moderate-to-vigorous physical activity and prevalence of achieving public health guidelines.

3. - Ham SA, Kruger J, Tudor-Locke C: Participation by US adults in sports, exercise, and recreational physical activities. $J$ Phys Act Health 2009, 6:6-14. This cross-sectional study described self-reported participation in sports, exercise, and recreational physical activity data collected from two national surveys of adults: the 2003-2005 American Time Use Survey (ATUS), and the 1999-2004 NHANES.

4. Murtagh EM, Boreham CA, Murphy MH: Speed and exercise intensity of recreational walkers. Prev Med 2002, 35:397-400.

5. •- Bravata DM, Smith-Spangler C, Sundaram V, et al.: Using pedometers to increase physical activity and improve health: a systematic review. JAMA 2007, 298:2296-2304. This systematic review analyzed eight randomized control trials and 18 observational studies and concluded that pedometer use is associated with significant improvements in physical activity, body mass index, and blood pressure.

6. •• Richardson CR, Newton TL, Abraham JJ, et al.: A metaanalysis of pedometer-based walking interventions and weight loss. Ann Fam Med 2008, 6:69-77. This meta-analysis identified nine randomized controlled trials and propsective cohort studies and reported modest weight loss with participation in pedometerbased walking programs.

7. •• Tudor-Locke C, Johnson WD, Katzmarzyk PT: Accelerometerdetermined steps/day in US adults. Med Sci Sports Exerc 2009, 41:1384-1391. This cross-sectional analysis of the 2005-2006 NHANES accelerometer data used a data manipulation to translate accelerometer data in terms of pedometer-based scales.

8. •• Tudor-Locke C, Bassett DR, Shipe M, McClain JJ: Pedometry methods for assessing free-living adults. J Phys Act Health 2010 (in press). This review updates pedometry methods in terms of instrument choice, metric choice, validity, reliability, data collection and retrieval, time worn, day-to-day variability, monitoring frame, reactivity, and data treatment.

9. Tudor-Locke CE, Myers AM: Methodological considerations for researchers and practitioners using pedometers to measure physical (ambulatory) activity. Res Q Exerc Sport 2001, 72:1-12. 
10. Crouter SE, Schneider PL, Karabulut M, Bassett DR Jr: Validity of 10 electronic pedometers for measuring steps, distance, and energy cost. Med Sci Sports Exerc 2003, 35:1455-1460.

11. Miller R, Brown W, Tudor-Locke C: But what about swimming and cycling? How to 'count' non-ambulatory activity when using pedometers to assess physical activity. $J$ Phys Act Health 2006, 3:257-266.

12. Tudor-Locke C, Williams JE, Reis JP, Pluto D: Utility of pedometers for assessing physical activity: construct validity. Sports Med 2004, 34:281-291.

13. Tudor-Locke C, Ainsworth BE, Whitt MC, et al.: Ambulatory activity and simple cardiorespiratory parameters at rest and submaximal exercise. Can J Appl Physiol 2003, 28:699-709.

14. - Cao ZB, Miyatake N, Higuchi M, et al.: Prediction of VO2max with daily step counts for Japanese adult women. Eur J Appl Physiol 2009, 105:289-296. This study developed and crossvalidated a model to predict $\mathrm{VO}_{2}$ max based on pedometerdetermined physical activity, age, and BMI.

15. - Schmidt MD, Cleland VJ, Shaw K, et al.: Cardiometabolic risk in younger and older adults across an index of ambulatory activity. Am J Prev Med 2009, 37:278-284. This cross-sectional analysis evaluated the utility of a pedometer-based physical activity index to discriminate cardiometabolic risk status.

16. - Savage PD, Ades PA: Pedometer step counts predict cardiac risk factors at entry to cardiac rehabilitation. J Cardiopulm Rehabil Prev 2008, 28:370-377. This cross-sectional study evaluated the correlation between pedometer-determined physical activity and cardiac risk factors in individuals beginning phase II cardiac rehabilitation.

17. Walsh JT, Charlesworth A, Andrews R, et al.: Relation of daily activity levels in patients with chronic heart failure to longterm prognosis. Am J Cardiol 1997, 79:1364-1369.

18. - Payvandi L, Dyer A, McPherson D, et al.: Physical activity during daily life and brachial artery flow-mediated dilation in peripheral arterial disease. Vasc Med 2009, 14:193-201. This cross-sectional study found that pedometer-determined physical activity was associated with brachial artery flow-mediated dilation in individuals with lower extremity peripheral arterial disease, even after adjusting for confounders.

19. Miyatake N, Nishikawa H, Morishita A, et al.: Daily walking reduces visceral adipose tissue areas and improves insulin resistance in Japanese obese subjects. Diabetes Res Clin Pract 2002, 58:101-107.

20. Sugiura H, Sugiura H, Kajima K, et al.: Effects of long-term moderate exercise and increase in number of daily steps on serum lipids in women: randomised controlled trial. $B M C$ Womens Health 2002, 2:3.

21. - McTiernan A, Sorensen B, Irwin ML, et al.: Exercise effect on weight and body fat in men and women. Obesity (Silver Spring) 2007, 15:1496-1512. This 12-month, randomized, controlled exercise trial used pedometers to evaluate changes in physical activity. Those with the greatest increases in steps/day achieved greater results in terms of weight, BMI, body fat, and intraabdominal fat.

22. Wyatt HR, Peters JC, Reed GW, et al.: A Colorado statewide survey of walking and its relation to excessive weight. $\mathrm{Med} \mathrm{Sci}$ Sports Exerc 2005, 37:724-730.

23. Tudor-Locke C, Ham SA, Macera CA, et al.: Descriptive epidemiology of pedometer-determined physical activity. Med Sci Sports Exerc 2004, 36:1567-1573.

24. Bassett DR, Schneider PL, Huntington GE: Physical activity in an Old Order Amish community. Med Sci Sports Exerc 2004, 36:79-85.

25. •- Marshall SJ, Levy SS, Tudor-Locke CE, et al.: Translating physical activity recommendations into a pedometer-based step goal: 3000 steps in 30 minutes. Am J Prev Med 2009, 36:410-415. This treadmill-based study collected pedometer steps and expired gases to determine steps/minute cut-points relative to moderate-intensity activity.

26. Tudor-Locke C, Sisson SB, Collova T, et al.: Pedometerdetermined step count guidelines for classifying walking intensity in a young ostensibly healthy population. Can J Appl Physiol 2005, 30:666-676.

27. •• U.S. Department of Health and Human Services: 2008 Physical Activity Guidelines for Americans: Be Active, Healthy, and Happy! Washington, DC: U.S. Department of Health and Human Services; 2008. Available at http://www.health.gov/paguidelines/ guidelines/default.aspx. These government-issued physical activity guidelines describe the types and amounts of physical activity that offer substantial health benefits.

28. Dunn AL, Andersen RE, Jakicic JM: Lifestyle physical activity interventions. History, short- and long-term effects, and recommendations. Am J Prev Med 1998, 15:398-412.

29. •• Tudor-Locke C, Johnson WD, Katzmarzyk PT: Relationship between accelerometer-determined steps/day and other accelerometer outputs in U.S. adults. J Phys Act Health 2010 (in press). This cross-sectional analysis of the 2005-2006 NHANES accelerometer data explored relationships between acclerometerdetermined steps/day and other accelerometer outputs, including estimates of time spent.

30. Jordan AN, Jurca GM, Tudor-Locke C, et al.: Pedometer indices for weekly physical activity recommendations in postmenopausal women. Med Sci Sports Exerc 2005, 37:1627-1632.

31. Tudor-Locke C, Bassett DR, Swartz AM, et al.: A preliminary study of one year of pedometer self-monitoring. Ann Behav Med 2004, 28:158-162.

32. Tudor-Locke C, Bassett DR Jr: How many steps/day are enough? Preliminary pedometer indices for public health. Sports Med 2004, 34:1-8.

33. •• Tudor-Locke C, Hatano Y, Pangrazi RP, Kang M: Revisiting "how many steps are enough?". Med Sci Sports Exerc 2008, 40 (Suppl 7):S537-S543. This article reviewed background information and updated scientific understanding of health-related walking doses expressed in terms of pedometer-determined physical activity for adults and youth.

34. Blair SN, Cheng Y, Holder JS: Is physical activity or physical fitness more important in defining health benefits? $\mathrm{Med} \mathrm{Sci}$ Sports Exerc 2001, 33(Suppl 6):S379-S399; discussion S419-S420.

35. - Tudor-Locke C: A short list about what I do and don't know: about objective monitoring of physical activity. Curr Sports Med Rep 2010, 9:71-72. This opinion piece reflects on current issues with regard to using objective monitors to monitor physical activity.

36. - Rothney MP, Apker GA, Song Y, Chen KY: Comparing the performance of three generations of ActiGraph accelerometers. J Appl Physiol 2008, 105:1091-1097. This validity study compared outputs from three different models of the same accelerometer brand using standardized mechanical oscillations.

37. - Kozey SL, Staudenmayer JW, Troiano RP, Freedson PS: A comparison of the ActiGraph 7164 and the ActiGraph GT1M during self-paced locomotion. Med Sci Sports Exerc 2009 (in press). This validity study compared outputs from two different models of the same accelerometer during self-paced walking at slow, medium, and fast speeds.

38. •• Tudor-Locke C, Lutes L: Why do pedometers work? A reflection upon the factors related to successfully increasing physical activity. Sports Med 2009, 39:981-993. This opinion paper explored characteristics of pedometers, pedometer-based physical activity interventions, and the participants who attend these programs to engage a better understanding of how to optimally use pedometers to facilitate successful behavior change.

39. Tudor-Locke C, Ainsworth BE, Thompson RW, Matthews CE: Comparison of pedometer and accelerometer measures of freeliving physical activity. Med Sci Sports Exerc 2002, 34:2045-2051. 